\title{
Estrategia de comunicación para la inserción laboral en Facebook. Grupos de empleo
}

\section{Communication strategy in Facebook for the entrance into the labour market. Employment groups}

\author{
Silvia Alende Castro \\ Doctoranda del departamento de Psicoloxía Evolutiva e \\ Comunicación de la Facultade de Ciencias Sociais e da Comunicación \\ de Pontevedra (Universidade de Vigo)
}

Fecha de recepción: 3 de octubre de 2014

Fecha de revisión: 12 de enero de 2015

Para citar este artículo: Alende Castro, S. (2015): Estrategia de comunicación para la inserción laboral en Facebook. Grupos de empleo, Icono 14, volumen (13), pp. 151-179. doi: 10.7195/ri14.v13i1.742 


\section{Resumen}

Este artículo pone en discusión resultados de la investigación Estrategia de comunicación para la inserción laboral en Facebook. Grupos de empleo. A nivel metodológico, este estudio se orientó hacia una perspectiva cuantitativa, a través del análisis de contenido y la realización de cuestionarios como técnicas de investigación. En el primer caso, el objeto de análisis fueron tres grupos vinculados al área del empleo en Facebook y correspondientes a otros tantos entornos territoriales de Galicia: autonómico, comarcal y municipal. En el segundo, fueron los usuarios de esas comunidades los destinatarios del citado cuestionario acerca de la utilidad de estos grupos y la propia red social para obtener un puesto de trabajo. Entre las conclusiones obtenidas, se puede afirmar que el funcionamiento de colectivos como los analizados se desarrolla a través de una estrategia de comunicación para la inserción laboral y articulada sobre una cultura colaborativa.

Palabras clave: Facebook - Grupos - Empleo - Comunicación - Cultura colaborativa - Inserción laboral

\section{Abstract}

This paper sets out of the research Communication strategy for the labour integration on Facebook. Employment groups. At the methodological level, this study was oriented towards a quantitative perspective, through the analysis of content and the completion of questionnaires as research techniques. In the first case, the object of analysis were three groups linked to the area of employment in Facebook and belonging to equal number territorial environments of Galicia: regional, local and municipal. In the second, were users of these communities the recipients of the questionnaire about the usefulness of these groups and the social network for employability. One of the conclusions that can be enunciated: the functioning of groups such as those tested develops a communication strategy for achieving labour integration and base on a collaborative culture.

Key Words: Facebook - Groups - Employment - Communication - Collaborative culture - Labour integration 


\section{Introducción}

\subsection{Plataformas cooperativas y redes sociales}

La reflexión sobre el influjo de los cambios tecnológicos en la comunicación, en particular en movimientos sociales y plataformas cooperativas, exige definir previamente los dos conceptos: movimientos sociales y plataformas cooperativas.

Algunos autores los incluyen en la categoría de actores políticos colectivos. Como tales, los movimientos sociales compartirían con partidos políticos o grupos de interés características como una relativa estabilidad organizativa, una comunidad de objetivos entre sus miembros, una línea de acción coordinada o la voluntad de intervenir en la gestión de conflictos (Martí i Puig, 2014).

En este contexto, pensamos que grupos vinculados al ámbito del empleo creados en la red social Facebook pueden ser entendidos como una plataforma cooperativa. Entendemos que la globalidad de esos grupos podría encuadrarse en esta categoría al tratarse de un nuevo espacio de participación en el que sus distintos miembros buscan unos objetivos de cambio en su situación laboral a través del mantenimiento de una línea de acción con continuidad en el tiempo (Martí i Puig, 2014).

El objeto de este trabajo se centra en el estudio de Facebook como herramienta para la inserción laboral. Este análisis se efectúa desde dos perspectivas: por una parte, se evalúa el uso que los propios usuarios hacen de la plataforma. Por otra, se tiene en cuenta el discurso y la comunicación que se establecen en esta red en relación a la temática laboral.

La literatura sobre la cuestión muestra que comprender los grupos de empleo en Facebook como plataforma cooperativa se aleja del tipo de movimientos sociales a los que estamos acostumbrados (más centrados en asuntos de calado político, ambiental o contrapoder). Sin embargo, el hecho de que respondan a otro tipo de temática y que su finalidad sea distinta no altera su consideración como espacio de participación generado a raíz de la evolución tecnológica. Como señaló -en línea con el pensamiento de Habermas- Claus Offe hace ya casi dos décadas, también en

DOI: ri14.v13i1.742 | ISSN: 1697-8293 | Año 2015 Volumen 13 Nº 1 | ICONO14 
este momento parece que "se está desdibujando la línea divisoria que deslinda los asuntos y comportamientos políticos de los privados" (1996: 163).

\subsection{Búsqueda de empleo en la esfera pública}

En la actualidad es visible cómo la búsqueda de empleo, antes relegada a un comportamiento íntimo e individual, ha dado un salto a la esfera pública. En medios como el que aquí se analiza, Facebook, se comprueba que esta acción ya no tiene solo carácter particular -propio de cada uno- sino que ha adquirido otros matices al convertirse en una actividad compartida. Castells sostiene que "la mayor parte de movimientos sociales y políticos del mundo de todas las tendencias utilizan Internet como una forma privilegiada de acción y de organización" (2000: 12).

Esta situación se produce en un período de transformaciones tecnológicas pero también de características concretas a nivel laboral. Al cierre del segundo trimestre de 2014, la tasa de paro se situó en España en un 24,47\%, según los resultados de la última Encuesta de Población Activa (EPA) publicada por el Instituto Nacional de Estadística (INE) para el mencionado período. Por comunidades autónomas, este mismo estudio señala una importante disminución del empleo en Galicia, con 13.900 ocupados menos a mediados de 2014 que en el año anterior. La región gallega se encuentra además entre las autonomías en las que más se enquista la búsqueda de empleo, al crecer en 4,8 puntos -desde el primer al segundo trimestre de 2014- el porcentaje de parados con dos o más años en situación de procura de un puesto de trabajo. En este contexto, no parece casual el uso de las redes como herramienta para la inserción laboral puesto que "Internet es la sociedad, expresa los procesos sociales, los intereses sociales, los valores sociales, las instituciones sociales" (Castells, 2000: 18).

Derivado del uso de Internet, el fenómeno de las redes sociales se acepta como una revolución en nuestro concepto de relación social clásica. Surgidas como un nuevo fenómeno tecnológico y social son "las grandes protagonistas actuales de la sociedad digital" (Caldevilla, 2010: 45) pese a que, compartiendo la idea de Juan Freire, "las redes forman parte de nuestra propia naturaleza biológica y cultural" puesto que "somos seres sociales y formamos redes desde nuestros inicios evoluti-

ICONO14 | Año 2015 Volumen $13 N^{\circ} 1$ | ISSN: 1697-8293 | DOI: ri14.v13i1.742 
vos" (2009: 585). El objetivo de estas nuevas plataformas es la creación de comunidades en línea mediante la representación de las conexiones principales que los usuarios disponen los unos de los otros (Ros-Martín, 2009: 555).

Otros autores convienen en proponer un marco común de conocimiento y entienden las redes sociales como "aquellas herramientas telemáticas de comunicación que tienen como base la Web, se organizan alrededor de perfiles personales o profesionales de los usuarios y tienen como objetivo conectar secuencialmente a los propietarios de dichos perfiles a través de categorías, grupos, etiquetados personales, etc., ligados a su propia persona o perfil profesional" (Castañeda y Gutiérrez, 2010).

Pese a parecer un fenómeno de reciente aparición, los antecedentes de las redes comienzan antes del nacimiento de la Web 2.0 (Marcos Ros-Martín, 2009: 555). En concreto, se acepta 1997 como la fecha de su creación con la inauguración de la web SixDegrees.com, una página que permitía activar perfiles, listados de amigos o navegar en listas de amigos. Hasta 2001, se asiste al surgimiento de numerosas herramientas de generación de perfiles o listado de amigos: AsianAve, BlackPlanet o MiGente, entre otras. En el año 2003, tras una crisis informática, nacieron las tres primeras redes sociales de internet: Tribe.net, LinKedin y Friendster. Con la explosión de la web 2.0., este tipo de sitios web comenzaron a proliferar y ganar en popularidad.

En el momento actual (2015), el uso de las redes sociales en España mantiene una tendencia creciente y se sitúa ya, según la Asociación para la Investigación de Medios de Comunicación (AIMC) -en su $16^{\text {a }}$ Encuesta a Usuarios de Internet-, en un 74,2\%. Es decir, 6,8 puntos por encima de los datos correspondientes al año anterior. La literatura recoge cuatro empleos generales, propuestos por Del Moral, de las redes sociales: mantenimiento de amistades, nueva creación de amistades, entretenimiento y gestión interna de organizaciones empresariales (Caldevilla, 2009: 52). Según el objeto que persigue el usuario de la red social, Inmaculada Berlanga distingue dos categorías distintas de redes sociales: de ocio -en las que prima el entretenimiento y las relaciones personales- y de uso profesional, encaminadas a la promoción de profesionales en un determinado campo o especialidad (2013: 89). 
En cuanto al modo en que se emplean estas webs, destaca el Smartphone como dispositivo para conectarse a la red social -el 85,5\% de los encuestados por AIMC lo usan con más frecuencia, durante más tiempo y para más usos- y el alza de conexión también desde la tablet -utilizada por un $43 \%$ de los usuarios-. Y en cuanto a la preferencia de las distintas redes por parte de los españoles, Facebook mantiene su hegemonía $(90,3 \%)$ entre los usuarios de este tipo de plataformas. Permanece estable el uso de Twitter $(45,1 \%)$ o de Linkedin $(24,2 \%)$. Entre las redes de mayor alza se encuentran Google+ $(37,7 \%)$ e Instagram (20,1\%).

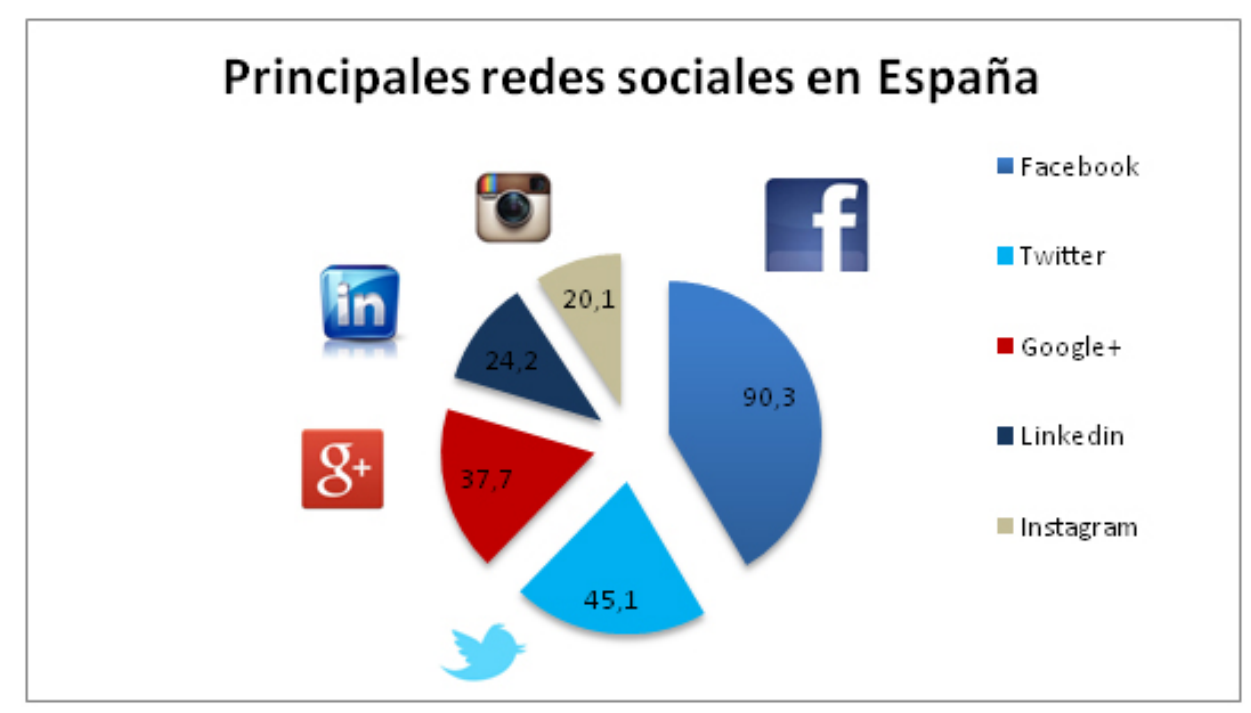

Figura 1: Empleo de las principales redes en España (2013)

Fuente: Elaboración propia a partir de AIMC

\subsection{Aplicativo grupos en Facebook}

Nacida en 2004 de la mano de Mark Zuckerberg como un sitio web para estudiantes de la Universidad de Harvard, en la actualidad Facebook ocupa el segundo puesto en el ranking mundial por el número de visitas recibidas, solo por detrás de Google, según los datos aportados por Alexa. La comparación por audiencia entre esta red social y las otras cuatro presenta a Facebook como paradigma de las redes sociales: 
Temas
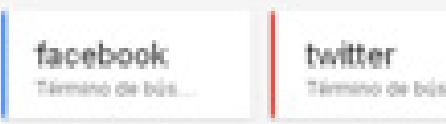

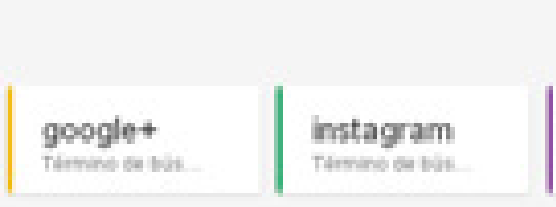

Emotate

Inkendn

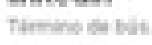

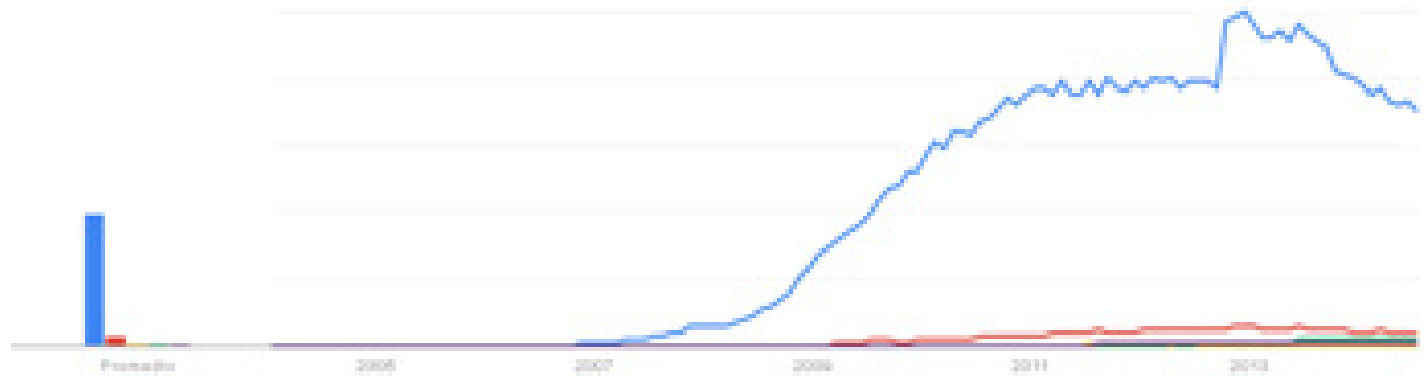

Figura 2: Comparativa de audiencia de redes sociales. Fuente: Google Trends

Informarse, expresarse y mantener conexión con el círculo social lejano son otras de las razones que llevan a los españoles a decantarse por esta plataforma, según The Coktail Analysis. Con unos 1.100 millones de usuarios registrados ${ }^{1}$, esta red social permite distintos modos de presencia. Además de crear perfiles (uso particular) o páginas (pensadas para corporaciones y empresas), otra de esas posibilidades es la de constituir grupos, unidad de análisis de esta investigación.

Resultan de interés las explicaciones ${ }^{2}$ al respecto que ofrece la propia compañía y según las cuales las páginas permiten a organizaciones, empresas, famosos y marcas reales comunicarse de forma general con la gente a la que gustan y solamente deben ser creadas y administradas por representantes oficiales. Sin embargo, los grupos proporcionan un espacio cerrado para que colectivos pequeños puedan intercambiar opiniones acerca de intereses comunes, de modo que cualquier persona puede crear un grupo. Las desigualdades más importantes entre ambas hacen referencia a la privacidad, el público o el tipo de comunicación: 


\begin{tabular}{|c|c|c|}
\hline & Página & Grupo \\
\hline Privacidad & $\begin{array}{c}\text { Información pública y disponible para } \\
\text { todo el grupo }\end{array}$ & $\begin{array}{l}\text { Además de la configuración abierta, } \\
\text { existen otras opciones de privacidad } \\
\text { disponibles para los grupos. } \\
\text { En grupos secretos y cerrados, las } \\
\text { publicaciones solamente están visibles } \\
\text { para los miembros del grupo }\end{array}$ \\
\hline Público & $\begin{array}{l}\text { No se limita el número de personas a } \\
\text { las que puede gustar una página }\end{array}$ & $\begin{array}{l}\text { Se puede modificar la privacidad del } \\
\text { grupo para que los miembros tengan } \\
\text { que ser aprobados o añadidos por los } \\
\text { administradores. Cuando un grupo } \\
\text { alcanza un determinado tamaño, se } \\
\text { comienzan a limitar algunas funciones }\end{array}$ \\
\hline Comunicación & $\begin{array}{c}\text { Las personas que ayuden a administrar } \\
\text { pueden compartir publicaciones desde } \\
\text { la página }\end{array}$ & $\begin{array}{l}\text { Los miembros reciben notificaciones } \\
\text { de forma predeterminada cuando algún } \\
\text { miembro publica una entrada en el } \\
\text { grupo. Los miembros del grupo pueden } \\
\text { participar en chats, subir fotos en los } \\
\text { álbumes compartidos, colaborar en } \\
\text { documentos del grupo e invitar a todos } \\
\text { los miembros que son amigos a eventos } \\
\text { del grupo }\end{array}$ \\
\hline
\end{tabular}

Tabla 1: Diferencias entre grupos y páginas de Facebook. Fuente: Facebook

Llorens y Capdeferro (2011: 36) señalan que, en el caso funcional de los grupos, la red social confiere soporte tecnológico inicial a los siguientes elementos: perfil de grupo (administrado por el creador o propietario del mismo), muro del grupo o tablón de los miembros, foros, fotos, vídeos y eventos. De modo genérico, en el funcionamiento de estas unidades se acepta que cuando se conceden permisos de administrador a los miembros del grupo, todos ellos pueden actuar sin restricciones en cualquiera de sus secciones excepto en los eventos, reservados a los propietarios. En función de su grado o nivel de visibilidad, los grupos pueden ser 
abiertos, privados o secretos. Los primeros son accesibles a todos los usuarios de la red social, los segundos pertenecen a una comunidad cerrada a la que se entra por invitación y los terceros no aparecen en los resultados de las búsquedas.

Entre los puntos fuertes de estos grupos, destacan la sencillez y rapidez de creación y administración, la simplicidad de uso de las herramientas, el chat, mensajería y taggind de imágenes, la capacidad de expansión interna, su potente soporte para el mobile learning y sus características añadidas de microblogging y lifestreaming (Llorens y Capdeferro, 2011: 36). A ellas pueden sumarse las posibilidades de promoción al invitar a contactos o la opción de los administradores de poder enviar mensajes a todos los miembros (Margaix-Arnal, 2008: 594). Al respecto, Marcelo Zamora indica: "intervenir en una red social empieza por hallar allí otros con quienes compartir nuestros intereses, preocupaciones o necesidades" (2006: 2).

Por otra parte, las desventajas hacen referencia a la moderación manual de mensajes y contactos, la vinculación directa del grupo con el administrador, la imposibilidad de enlace a blogs y Twitter o la ausencia de estadísticas a la vista (García, 2010). En sentido genérico, se entiende por grupo a una reunión de dos o más individuos con relación entre sí y con, al menos, un objetivo en común. De hecho, es la certeza de que juntos pueden tratar de alcanzar esa meta en mejores condiciones de lo que lo harían individualmente lo que los hace actuar en conjunto (Acosta, 2012: 689).

Con la noción de contrato que propone Charaudeau (2006) como marco, Acosta señala que el aplicativo grupos en Facebook "constituye un nivel global de contrato comunicativo" (2012: 697) en el sentido de que todos los usuarios han de conocer las normas psicosociales y discursivas para operar en el mismo. Esta autora propone distintos modos de apropiación -usos- que los usuarios hacen de estos grupos en función de variables entre las que se estudian la finalidad, la identidad de los participantes o los propósitos de cada grupo: Estrategia de la comunicación pública y política, Estrategia de la comunicación publicitaria y del mercadeo, Estrategia de gestión de la comunicación organizacional, Estrategia para la gestión de la producción artística, cultural y académica, Medio para la construcción de sociabilidades e identidades, Escenario de ocio y diversión y Escenario de participación social y política: micropolítica (Acosta, 2012: 697).

DOI: ri14.v13i1.742 | ISSN: 1697-8293 | Año 2015 Volumen 13 Nº 1 | ICONO14 
En esta investigación, a esta tipología abierta podría sumarse un nuevo modo de apropiación que proponemos denominar Estrategia de la comunicación para la inserción laboral. En este uso se englobarían aquellos grupos de la red social cuyas acciones se orienten a la consecución de la inserción laboral de sus integrantes. Su finalidad sería la promoción de la empleabilidad en un espacio de participación en el que no hay, obligatoriamente, diferencia de roles entre emisor y receptor sino que cualquiera de los miembros del colectivo puede ocupar ambos papeles.

Más allá de las acciones concretas que puedan desarrollarse en el seno de grupos de inserción laboral como los que se analizan en este trabajo, subyace la noción de cultura colaborativa como el concepto que articularía su funcionamiento. Es decir, las redes sociales y en particular los grupos en torno a un objetivo compartido constituirían nuevas plataformas que facilitan la comunicación, el contacto o la difusión de información de interés para la colectividad. De esta manera, "se fomenta una idea de comunidad [...] y se favorece la cultura de la colaboración" (Arroyo-Vázquez, 2009: 146).

Aprovechar las redes de contactos que gestionan los grupos de Facebook supone un modo de demostrada eficacia para distintas gestiones de la realidad presencial. Informar, solicitar apoyo y ayuda o mostrar expectativas constituyen modalidades encaminadas la consecución de determinados objetivos, proceso conocido como Networking. En esta línea, las redes sociales "han dotado a este mecanismo ancestral de la inmediatez, la transparencia y la mundalización que les caracteriza" (Castañeda y Gutiérrez, 2010).

En la actualidad, ese entorno se multiplica exponencialmente, extendiéndose a otros contactos en un radio espacial tan amplio como la red y en un corto período de tiempo. Apunta Ros-Martín que la teoría germinal del desarrollo de plataformas para la creación de redes sociales parte de la teoría de los Seis Grados de Separación, expuesta en 1929 por el escritor Frigyes Karinthy y según la cual el número de conocidos de una persona crece exponencialmente siguiendo un número de enlaces de una cadena que serían las relaciones humanas $(2009,553)$. En este nuevo escenario, cabe preguntarse entonces si las redes sociales -y en particular los grupos de empleo- abren un nuevo mecanismo y un amplio abanico de posibilidades al

ICONO14 | Año 2015 Volumen $13 N^{\circ} 1$ | ISSN: 1697-8293 | DOI: ri14.v13i1.742 
mundo laboral. El uso masivo de Facebook como red social por antonomasia permite ver ya a esta web como plataforma de puesta en contacto de profesionales de muy distintos ámbitos. Esta investigación ofrece resultados cuantitativos sobre su uso como plataforma para acceder a puestos de trabajo.

\section{Material y Métodos}

Este estudio parte de un presupuesto inicial según el cual, en la actualidad, las redes sociales podrían entenderse como seno de un nuevo movimiento social para el fomento, en red, de la inserción laboral. Es decir, se propone analizar si el funcionamiento de grupos en la red social Facebook centrados en el intercambio de información laboral supone un nuevo modo de cooperación, entre todos los miembros del colectivo, frente a situaciones de desempleo. Para ver si efectivamente esto puede entenderse así se plantean las siguientes hipótesis:

H1: La cultura colaborativa es la base del funcionamiento de los grupos de empleo en Facebook, pues en ellos prima la difusión de información de oferta de empleo sobre la promoción personal o de candidaturas individuales.

H2: Por uso y grado de actualización, los grupos de empleo en Facebook pueden ser considerados herramientas de comunicación para el fomento de la inserción laboral dado que son instrumentos de recurrencia diaria y renovación constante de contenidos.

H3: La información sobre vacantes es la que recibe un mayor apoyo por parte del colectivo.

Para poder comprobar o refutar estas tres hipótesis, se ha optado por una metodología cuantitativa (Hernández Sampieri, 2010: 34) conformada por dos técnicas: el análisis de contenido y el cuestionario. En primer lugar, se seleccionó una muestra integrada por tres grupos de esta red social centrados en temática laboral y referentes, en concreto, a Galicia (comunidad, como se señaló antes, con características propias respecto a la situación laboral): Ofertas de trabajo en Galicia ${ }^{3}$, Oferta y demanda de empleo en Santiago de Compostela y comarca ${ }^{4}$ y Ofertas e demandas

DOI: ri14.v13i1.742 | ISSN: 1697-8293 | Año 2015 Volumen 13 Nº 1 | ICONO14 
de emprego na Estrada ${ }^{5}$. Con esta selección se buscaba que los resultados de este estudio fueran referentes además a tres ámbitos territoriales: el autonómico, el comarcal y el municipal, respectivamente. Cabe matizar que aunque en el primer caso (Ofertas de trabajo en Galicia) su denominación hace referencia únicamente a la publicación de ofertas de empleo, las normas del colectivo permiten también la promoción personal de los candidatos a través de la difusión de currículos, por lo que se consideró una comunidad apta para este estudio.

Estos tres grupos fueron sometidos a un análisis de contenido para el que se tomaron datos en la semana del 16 al 21 de junio, estableciéndose los siguientes días de recopilación de material: lunes 16, miércoles 18 y viernes 20. A continuación, se aplicó a estos colectivos el denominado online communication model, una metodología propuesta por Juan Narbona (2012) como marco para el estudio de la comunicación en internet. Se trata de un método de análisis de contenido ideado para el análisis de redes sociales que se distribuye en tres grandes bloques de análisis: nivel de persuasión, nivel de usabilidad y nivel de interactividad. El primero de estos bloques incluye las variables identificación, actualidad, papel del administrador, relevancia y fuentes. En usabilidad, se estudian las categorías aplicaciones, exclusividad de los contenidos y multimedialidad. Finalmente, el nivel de interactividad hace referencia a apoyos, comentarios, intervención y diálogo. Así, en el primer nivel se valora el tipo de actividad comunicativa que se produce en estas plataformas -el qué se hace-, en el segundo los recursos a través de los que se produce esta actividad -los medios a través de los que se hace- $\mathrm{y}$ en el tercero el modo en que se produce esa comunicación, es decir, el cómo.

La metodología de investigación se completó con la distribución, entre los miembros de cada grupo, de un cuestionario de elaboración propia con el que a través del planteamiento de 21 preguntas de respuesta única seleccionable entre una lista propuesta de opciones y una de respuesta tipo párrafo- se buscó obtener información de primera mano sobre el uso que los integrantes hacen del grupo, el perfil de cada usuario, sus principales acciones y su nivel de satisfacción general. 


\section{Resultados: Análisis por niveles y cuestionarios}

\subsection{Análisis de contenido}

El análisis de contenido aplicado a los tres grupos de la red social Facebook que conforman la muestra de este estudio arroja resultados en tres niveles: persuasión, usabilidad e interactividad.

\section{Nivel de persuasión}

En este nivel, se valoraron las siguientes variables: identificación, actualidad, papel del administrador, relevancia y fuentes. En relación a la categoría que encabeza el nivel de persuasión, esta investigación muestra que los colectivos Ofertas de trabajo en Galicia, Oferta y demanda de empleo en Santiago de Compostela y comarca y Ofertas e demandas de emprego na Estrada presentan una completa identificación compuesta, en todos los casos, por imagen de portada, nombre del grupo y presentación del mismo, url, normas de uso, identificación de los administradores y posibilidad de contacto.

Es en la variable actualidad, que mide el nivel de actividad, donde comienzan a apreciarse diferencias entre los colectivos estudiados. Así, con 33,3 post por día (100 en las tres jornadas de recolección de los datos) Ofertas de trabajo en Galicia se presenta como la comunidad con un mayor nivel de actualización, seguida de Ofertas e demandas de emprego na Estrada con 11,6 (35 en total) y Oferta y demanda de empleo en Santiago de Compostela y comarca, que alcanzó una media de 4,3 publicaciones diarias (13 en 3 días). A la luz de estos datos se comprueba que un supuesto ámbito de cobertura mayor no supone un mayor índice de actividad. En este caso, el colectivo comarcal (Oferta y demanda de empleo en Santiago de Compostela y comarca) presenta un grado de actualización menor al municipal (Ofertas e demandas de emprego na Estrada) pese a que se le presupone un mayor índice de usuarios con interés en el empleo que puede generarse en la zona, correspondiente además a la capital de la comunidad autónoma.

El período de análisis ayudó a comprobar que se trata de grupos con actividad diaria y constante, hecho que permite empezar a corroborar la veracidad de una de las hipótesis de este estudio -por uso y grado de actualización, los grupos de em-

DOI: ri14.v13i1.742 | ISSN: 1697-8293 | Año 2015 Volumen 13 Nº 1 | ICONO14 
pleo en Facebook pueden ser considerados herramientas de comunicación para el fomento de la inserción laboral. Son instrumentos de recurrencia diaria y renovación constante de contenidos- en la primer parte de su formulación. En el momento de análisis del uso que los integrantes hacen de estos colectivos se observará si este supuesto puede darse completamente por cierto.

El valor de actualidad tampoco sigue un patrón proporcional al número de miembros del colectivo. Ofertas de trabajo en Galicia es el grupo más numeroso y también el de mayor actualización (28.561 integrantes en el momento de recopilación de estos datos). Oferta y demanda de empleo en Santiago de Compostela y comarca (6.374) es el colectivo de menor actividad. Y el grupo más reducido, Ofertas e demandas de emprego na Estrada (1.515) ocupa la segunda posición por indice de actualización.

En relación al papel del administrador, en los dos grupos con un índice de actualización más elevado, Ofertas e demandas de emprego na Estrada y Ofertas de trabajo en Galicia, la actividad de esta figura es porcentualmente más importante. Destaca en el primero, en el que el $94 \%$ de los post emitidos en los días de análisis fue publicado por alguno de sus tres administradores. En el caso de la comunidad a nivel autonómico, esto ocurre en casi una de cada dos publicaciones, alcanzando el papel del creador del grupo un valor del $47 \%$. Finalmente, en Oferta y demanda de empleo en Santiago de Compostela y comarca la actualización corresponde completamente a los usuarios sin derechos de administrador, figura que en el período estudiado no emitió ningún post.

En la variable relevancia, la metodología propuesta por Narbona incluye dos cuestiones distintas pero ligadas entre sí: por una parte el tipo de temática a que corresponde cada publicación y, por otra, la medición del apoyo recibido por cada una de estas áreas. En este caso, se partía de los siguientes campos temáticos: vacantes, otra información laboral, búsqueda de colaboradores, boletines de empleo, testimonios de demandantes, oferta de servicios de formación, contexto, petición de ayuda, ánimo de lucro y otros. Pese a tratarse de ámbitos que, a priori, se contaban como de posible interés en este tipo de grupos, concluido el análisis se comprobó la inexistencia de algunos de ellos.

ICONO14 | Año 2015 Volumen 13 Nº 1 | ISSN: 1697-8293 | DOI: ri14.v13i1.742 
Así, puede afirmarse que los post relativos a la categoría temática Vacantes son, de modo genérico, los de mayor importancia numérica en los tres casos analizados. En Ofertas e demandas de emprego na Estrada y Ofertas de trabajo en Galicia representan casi la totalidad de los post emitidos (89\%). En Oferta y demanda de empleo en Santiago de Compostela y comarca suponen un 54\%. Al medir la relevancia -cuantificando y calculando el porcentaje de "me gusta" recibidos por los post de cada categoría- los usuarios de las tres comunidades vuelven a asimilarse al alcanzar, respectivamente, los siguientes valores: $100 \%$, $89 \%$ y $86 \%$. Los datos permiten observar entonces que la información sobre vacantes es la que recibe la mayor parte de los apoyos dados a las publicaciones en los tres grupos, tal y como se presuponía -y después se pudo comprobar- en la tercera hipótesis de partida, que establecía que la información sobre vacantes era la que recibía un mayor apoyo por parte del colectivo.

Entre sus objetivos, este estudio se proponía reflexionar también acerca del uso de los grupos de empleo como herramienta para la promoción de candidaturas laborales. Por este motivo, entre las categorías temáticas propuestas se incluyó una relativa a la presencia de Testimonios de demandantes de empleo en la que se tuvo en cuenta el contenido publicado para presentar profesionales, para la promoción de aptitudes propias o difundir currículums, entre otros. Sólo en dos de los grupos se detectó esta información. En Oferta y demanda de empleo en Santiago de Compostela y comarca es donde con más frecuencia se utiliza el muro del grupo para promover demandas de empleo. En este colectivo, la categoría temática Testimonios de demandantes acumula un $23 \%$ de la totalidad de post en el período analizado y un $17 \%$ de los "me gusta" totales recibidos. Su presencia es mínima en Ofertas de trabajo en Galicia (1\%) e inexistente en Ofertas e demandas de emprego na Estrada. Se comprueba así la hipótesis de partida de esta investigación, según la cual en el uso de grupos de temática laboral en Facebook prima la difusión de información de oferta de empleo sobre la promoción personal o de candidaturas personales. Por tanto, se observa cómo el funcionamiento de los grupos de empleo en Facebook se articula en torno a una cultura colaborativa que fomenta la ayuda entre todos sus integrantes más que en el uso individualista o personal de los mismos.

DOI: ri14.v13i1.742 | ISSN: 1697-8293 | Año 2015 Volumen 13 Nº 1 | ICONO14 


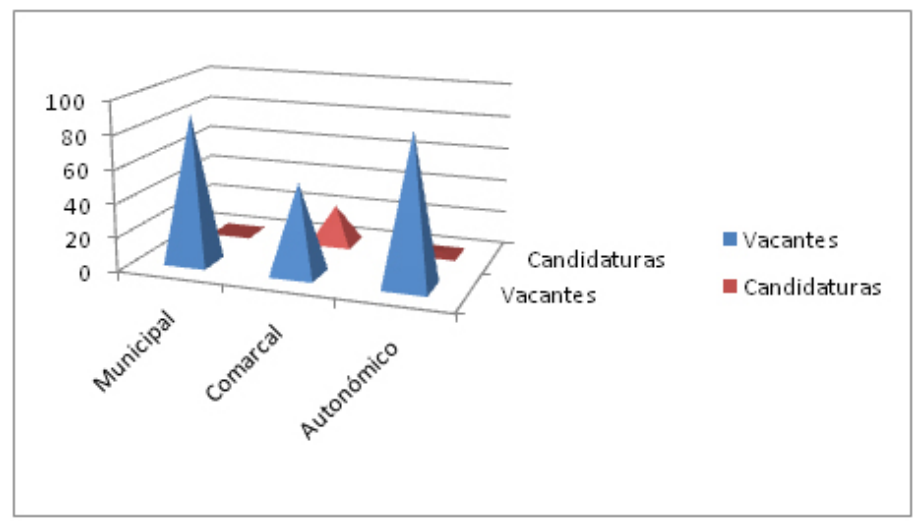

Figura 3: Usos de grupos de empleo Fuente: Elaboración propia

Entre las demás categorías temáticas propuestas, solo Ofertas de servicio de formación aparece en los tres grupos analizados. Porcentualmente, supone un $11 \%$ de los post de Ofertas e demandas de emprego na Estrada, un 8\% de Oferta y demanda de empleo en Santiago de Compostela y comarca y solo un $1 \%$ en Ofertas de trabajo en Galicia. Además de estas áreas, en el grupo comarcal pudo observarse la presencia de contenidos del tipo Otra información laboral, ámbito en el que se enmarca un $15 \%$ de los post analizados y no se cuantificó ningún apoyo. Por su parte, en el colectivo de carácter autonómico se detectó un 7\% de post relativos a Boletines de empleo, un $1 \%$ de Otra información laboral y un 1\% de Contexto.

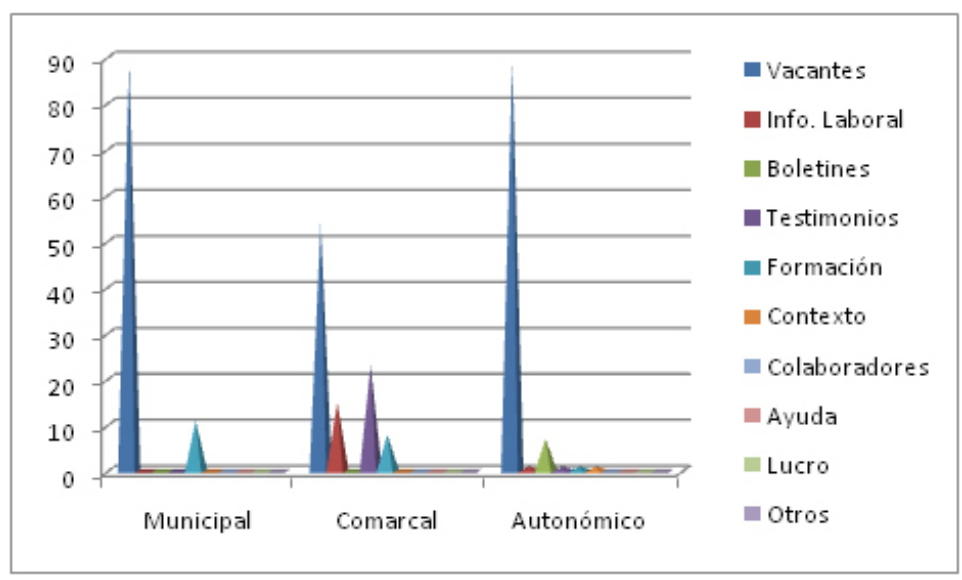

Figura 4: Presencia de contenidos por categorías Fuente: Elaboración propia 
El análisis del tipo de fuentes empleadas en estos grupos cerró el estudio del nivel de persuasión. En este caso, se observa cómo en dos de los grupos, Ofertas de trabajo en Galicia y Ofertas e demandas de emprego na Estrada, la práctica totalidad -un $88 \%$ y un $83 \%$ respectivamente- de los contenidos publicados en los muros de ambos colectivos se corresponden a post de alguna entidad de empleo compartidos por los usuarios de la comunidad. En Oferta y demanda de empleo en Santiago de Compostela y comarca esta fuente alcanza un valor del $31 \%$. En este último caso, a diferencia de los dos anteriores, es el contenido original el que, con un 54\%, prima sobre los demás (en los que supone solo, en cada uno de ellos, un 3\% del total) mientras que los post compartidos de entidades de empleo son el 31\% del total. Entre las demás fuentes que se han detectado figuran otras instituciones (un $8 \%$ en el caso del grupo municipal y un $6 \%$ en el autonómico) y también empresas (6 en la comunidad municipal, 15, 3\% en la comarcal y $3 \%$ en la autonómica).

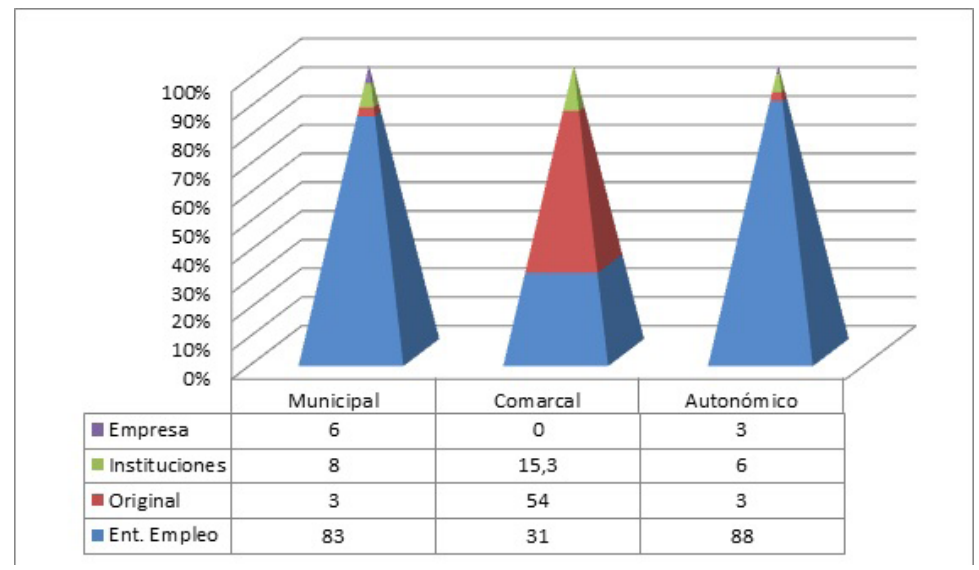

Figura 5: Fuentes de origen de contenidos Fuente: Elaboración propia

\section{Nivel de usabilidad}

Tras el nivel de persuasión, se tuvo en cuenta para cada grupo su índice de usabilidad, compuesto por tres nuevas variables: aplicaciones, exclusividad de los contenidos y multimedialidad. A través de la categoría Aplicaciones, se pudo sistematizar cuáles son los distintos tipos de elementos empleados por los grupos de empleo para la difusión de contenido. En este sentido, quedó comprobado el uso 
de enlaces y fotografías en Ofertas e demandas de emprego na Estrada y Oferta y demanda de empleo en Santiago de Compostela y comarca así como de ambos instrumentos junto con documentos (Word o PDF) en Ofertas de trabajo en Galicia. Esta variable se relaciona estrechamente con la tercera del nivel de usabilidad: multimedialidad. Además de valorar el modelo de aplicación utilizado ofrece resultados cuantitativos sobre esta cuestión. Según los datos recogidos, el índice de multimedialidad es del $94 \%$, el $66 \%$ y el $99 \%$ respectivamente.

\section{Nivel de interactividad}

Finalmente, el nivel de interactividad busca -a través de las variables apoyos, comentarios, intervención y diálogo- ver cómo es la relación que se produce entre los distintos miembros de cada grupo. Para valorar, en primer lugar, el índice de apoyo de la comunidad se realiza un cociente entre el total de apoyos ("me gusta") recibidos por los post publicados en el período de análisis y el número de integrantes de cada colectivo. De esta operación se deduce que la tasa de apoyo es, en los tres casos, reducida. El grupo que presenta un mayor apoyo a sus publicaciones es Ofertas de trabajo en Galicia, cuyo nivel es de 0,006 al cuantificarse 191 "me gusta" por parte de sus 28.561 miembros. Le sigue Ofertas e demandas de emprego na Estrada, colectivo integrado por 1.515 usuarios que, en las tres jornadas de estudio, realizaron 9 "me gusta", lo que supone una tasa de apoyo de 0,005. Aunque el número absoluto de "me gusta", 12, es mayor en Oferta y demanda de empleo en Santiago de Compostela que en el colectivo municipal, la tasa de apoyo es la más baja de los tres al contar con un número de integrantes mayor: 6.374. De ello resulta, para este último caso, un nivel de 0,002.

Otro de los elementos estudiados para el análisis de la interactividad es la cantidad de comentarios recibidos por los post analizados, dato empleado tanto en la variable Comentarios como en Intervención. En este caso, de nuevo se observa que la posibilidad de comentar es un recurso poco empleado. Aunque esta situación se produce, de modo generalizado, en los tres grupos que conforman la muestra de análisis es más visible en Ofertas e demandas de emprego na Estrada, en el que no se registró ningún comentario. Por su parte, en Oferta y demanda de empleo en Santiago de Compostela se cuantificaron dos, lo que supone una media de 0,15 comentarios por post. Finalmente, con un promedio de 0,21 y 21 comentarios,

ICONO14 | Año 2015 Volumen 13 Nº 1 | ISSN: 1697-8293 | DOI: ri14.v13i1.742 
Ofertas de trabajo en Galicia es el colectivo, de los tres, con una tasa de comentarios mayor.

Finalmente, al observar -en la variable Diálogo- si los administradores del grupo intervienen en algún momento en las escasas conversaciones generadas se pudo comprobar que en ningún momento se generó diálogo entre ellos al no cuantificarse ningún comentario emitido por el propietario o creador.

\subsection{Cuestionarios}

Como se apuntó anteriormente, al análisis de contenido ahora detallado se sumó la realización de un cuestionario cuyos resultados ayudaron a comprobar o reforzar las hipótesis de partida. De elaboración propia, este instrumento titulado $¿ E s$ Facebook una herramienta útil para la inserción laboral? se distribuyó entre los integrantes de los tres grupos de estudio. Con intención de evitar el influjo o sesgo del investigador que pudiese llegar a producirse, se optó por contactar con los administradores de cada uno de los colectivos y solicitar que fuesen ellos los que hiciesen público el cuestionario en el muro de cada grupo. En total, entre los días 3 y 13 de julio -período de activación del mismo- se recibieron 54 respuestas, de las que fueron válidas 52 al establecerse la obligatoriedad de respuesta afirmativa en la pregunta inicial ¿Hace uso de un grupo de empleo actualmente?

Los resultados obtenidos se exponen, a continuación, en seis bloques: acceso (mide la frecuencia de uso del grupo por parte de sus usuarios), perfil (situación y objetivo laboral de los usuarios), pertenencia (tiempo de agregación de los integrantes al grupo, modo en que se tuvo conocimiento de la existencia de la plataforma y razones de adhesión al mismo), uso (intención y acciones desarrolladas en el grupo), promoción de candidaturas personales (empleo del grupo para la presentación y difusión de perfiles profesionales) y satisfacción (valoración global de la plataforma).

\section{Acceso}

En primer lugar, se solicitó respuesta a tres cuestiones ideadas con la finalidad de medir la frecuencia de acceso y el tiempo de uso del grupo de empleo por parte

DOI: ri14.v13i1.742 | ISSN: 1697-8293 | Año 2015 Volumen 13 Nº 1 | ICONO14 
de cada usuario. En este sentido, un 73\% de los encuestados señaló que había accedido por última vez al muro de colectivo en la propia jornada de realización del cuestionario, resultado que indica un uso actual del grupo, que otro $10 \%$ consultó durante la semana de respuesta.

Casi 8 de cada 10 encuestados (un 78\%) señalaron que su acceso al grupo se produce a diario. De los usuarios con acceso diario a un grupo de empleo, el 46\% entra entre 1 y 2 veces al día. También es significativo, sin embargo, el 30\% de usuarios que confiesa acceder entre 304 veces en una misma jornada así como el 15\% que lo utiliza 5 o más veces en un día y el $9 \%$ que señala un uso de 100 más ocasiones por jornada. Por tanto, con estos datos en detalle del acceso de los usuarios al grupo quedó comprobada la veracidad de la segunda hipótesis de este estudio, según la cual por uso y grado de actualización, los grupos de empleo en Facebook pueden ser considerados herramientas de comunicación para el fomento de la inserción laboral al tratarse de instrumentos de recurrencia diaria y renovación constante de contenidos.

En relación al modo de acceso a estos colectivos, 9 de cada 10 usuarios se decantan por el ordenador o el teléfono móvil. En concreto, un 49\% de los encuestados señalan el PC como el principal dispositivo tecnológico que emplean para conocer las actualizaciones del grupo mientras que el smartphone es el instrumento preferido por un $38 \%$. La tablet, por su parte, es escogida por un $12 \%$ de los integrantes consultados.

Tras estas cuestiones iniciales, el cuestionario incluyó otras 17 preguntas que, aun presentándose distribuidas aleatoriamente por el mismo, hacen referencia a los restantes bloques mencionados: perfil de los usuarios, pertenencia de los mismos al colectivo, uso de la plataforma, promoción de candidaturas personales y satisfacción.

\section{Perfil}

En relación al primero de estos asuntos, el perfil, un 71\% de los participantes señaló ser desempleado en el momento de respuesta al mismo mientras que tan solo un $20 \%$ apuntó estar en activo, un 18\% como empleado y un $2 \%$ como trabaja-

ICONO14 | Año 2015 Volumen 13 Nº 1 | ISSN: 1697-8293 | DOI: ri14.v13i1.742 
dor por cuenta propia. En este sentido, no sorprende que el fin de la participación de un $76 \%$ de los usuarios sea conseguir un empleo mientras un $20 \%$ buscarían mejorar el actual. Entre los métodos para la consulta de información laboral, casi 9 de cada 10 encuestados (un 88\%) apuntan a internet como su fuente primordial. De las distintas fuentes de información laboral, un $42 \%$ de los usuarios preguntados reconoce que un grupo de empleo en Facebook se encuentra ya entre su principal vía de recepción de este tipo de contenido.

\section{Pertenencia}

Cuestionados por su pertenencia a uno de estos colectivos, casi la mitad de los encuestados (un 42\%) calculó entre seis meses y un año su tiempo como integrante de un grupo de empleo en la red social. Del mismo modo, un 40\% señaló ser miembro desde hacía, en el momento de realización del cuestionario, menos de seis meses. Para el 13\% este período es de uno a dos años mientras el $4 \%$ se registró hace ya más de dos años.

Tras esta cuestión temporal, se preguntó por el modo en el que los usuarios habían conocido el grupo. A esta interrogación, el 47\% respondió haber tenido constancia del mismo a través de sugerencias de la propia red social mientras que para el $42 \%$ fue una recomendación de amigos el motivo que les llevó a conocer dicha existencia. Un $11 \%$ indicó razones correspondientes a la opción 0tro.

Con intención de que fuesen los propios consultados los que pudiesen reflexionar y profundizar en los motivos que los llevaron a registrarse en un grupo de empleo en Facebook se ofreció, en esta cuestión, la fórmula de párrafo libre como modalidad de respuesta. Los datos obtenidos se consideran de gran valor precisamente por recoger, gracias a este modelo, numerosos matices informativos que una lista de opciones preconcebidas podría haber dejado fuera. Así, las razones indicadas con mayor frecuencia son las relativas a buscar empleo, estar desempleado o estar al tanto de ofertas. Sin embargo, a nivel cualitativo se consideran también de gran valía otras aportaciones.

Entre las vinculadas a la consulta de información, se recibieron respuestas como ver cómo está el panorama laboral en mi zona, estar al día de todas las

DOI: ri14.v13i1.742 | ISSN: 1697-8293 | Año 2015 Volumen 13 Nº 1 | ICONO14 
ofertas de trabajo y sobre todo de las que no se publican en los grandes portales de empleo, facilitan los boletines de empleo y al mismo tiempo informan de ofertas que salen publicadas en carteles en locales, así es más fácil que no se te escape ninguna (oferta) o tenemos más posibilidades de encontrarlo (un empleo). Asimismo, otro de los campos destacados entre las respuestas obtenidas es el de la ayuda y la colaboración entre todos para facilitar tanto la propia inserción laboral como la ajena: compartir, entre todos nos ayudamos subiendo las ofertas de empleo que encontramos y aunque no sea apto para nosotros siempre hay alguien a quien le puede venir bien, para buscar a amigos y familiares, ayudar a la máxima gente posible, porque mi marido está sin trabajo o para hacer networking. Finalmente, otro de los aspectos que llama la atención entre las 52 respuestas logradas es el referente a la facilidad de consulta y acceso a la información laboral: es una forma, desde mi punto de vista, más rápida y eficaz, por la rapidez, es más fácil y cómodo o alternativa a la habitual del boca a boca o de presentar c.v.

\section{Uso}

Además de conocer el perfil de los usuarios y las características de su pertenencia a un grupo de empleo, el cuestionario formulado permitió obtener detalles del uso que se hace de esta plataforma. De este modo, la primera pregunta de este bloque ¿con qué intención principal accede al grupo? ofreció respuestas que permitieron reforzar la veracidad, ya comprobada en el análisis de contenido, de la hipótesis principal puesto que casi la mitad de los miembros del colectivo, un 44\%, señaló que recibir información sobre vacantes era la principal finalidad de su pertenencia al mismo mientras que la promoción personal a través de la presentación de candidaturas solo es el objetivo de mayor importancia para el $16 \%$ de los consultados. Sobre esta intención priman otras como son estar al tanto de boletines de empleo (26\%) mientras que conocer otra información laboral supone la acción más trascendente en un $13 \%$ de los casos.

Se solicitó a los usuarios que accedieron a realizar el cuestionario que señalasen, de entre una lista de opciones, la que identificaban como más habitual en su caso. A ello el $81 \%$ señaló consultar información y un 4\% publicarla mientras que un $15 \%$ reconoció efectuar ambas. Dada la importancia que se le presuponía

ICONO14 | Año 2015 Volumen 13 Nº 1 | ISSN: 1697-8293 | DOI: ri14.v13i1.742 
-y después se corroboró- a la consulta como acción destacada dentro del muro se indagó, a continuación, en las actividades que conformaban esta acción concreta. En este caso, las respuestas se polarizaron en dos grandes opciones: solo consulto fue la posibilidad seleccionada por el $31 \%$ de los encuestados mientras que otro $25 \%$ confirmó ser usuarios con una mayor actividad al decantarse por la opción consulto, "me gusta", comento y comparto. Los datos reflejan por tanto la existencia, en el seno de estos colectivos, de dos grandes roles de usuarios: pasivos y activos. Otro $22 \%$ señaló que su acción habitual se corresponde a consulto y apoyo a través de "me gusta".

\section{Promoción de candidaturas personales}

Tras conocer cómo definen los usuarios su propia actividad dentro de la comunidad online, el cuestionario se encaminó hacia el ámbito de la utilización del mismo como herramienta para la promoción de candidaturas personales. Para ello, se formuló una pregunta directa en la que se buscaba conocer qué porcentaje de integrantes de la misma la habían empleado para presentar su perfil profesional. El dato obtenido fue del 25\%. Es decir, casi 8 de cada 10 usuarios miembros de un grupo de empleo nunca habían usado el mismo para su promoción personal. Sin embargo, preguntados por la rentabilidad obtenida el 75\% reconoce que sí había accedido a ofertas de empleo a través de este colectivo y casi la totalidad de los consultados, el 92\%, confiesa que si tuviese la seguridad de que el grupo es consultado por empresas que buscan candidatos sí presentaría su perfil profesional públicamente.

\section{Satisfacción}

Finalmente, el cuestionario abordó el nivel de satisfacción de los miembros de grupos de empleo en Facebook. En general, el 44\% de los mismos se confesó más bien satisfecho, un $21 \%$ sumamente satisfecho y un $23 \%$ ni insatisfecho ni satisfecho. Como sumamente o más bien insatisfechos se mostraron el 4 y el $8 \%$ de los preguntados, respectivamente. Por tanto, puede afirmarse que el grado de contento de sus usuarios con los grupos de empleo es alto, extremo que reafirma el hecho de que un $94 \%$ de los consultados apunte que recomendaría a un amigo que se hiciese miembro de un colectivo de este tipo.

DOI: ri14.v13i1.742 | ISSN: 1697-8293 | Año 2015 Volumen 13 Nº 1 | ICONO14 


\section{Discusión}

\subsection{Verificación de hipótesis}

A la luz de los datos arrojados por la metodología empleada en este estudio, se puede afirmar la veracidad de los supuestos que, desde un primer momento, conformaron el punto de partida de esta investigación. En relación a la primera hipótesis de este estudio, se ha comprobado que la cultura colaborativa es la base del funcionamiento de los grupos de empleo en Facebook, al tiempo que se confirmó que, en el seno de estos colectivos, la difusión de oferta de empleo prima sobre su uso como método de promoción personal o de candidaturas individuales. Como se pudo demostrar, los post pertenecientes a la categoría Vacantes son los más frecuentes y también los más apoyados por los usuarios -tal y como se establecía en la tercera hipótesis de investigación-, posicionándose a gran distancia -tanto en número como en nivel de apoyo- de los contenidos relativos a demanda de empleo o promoción de candidaturas personales. Al tiempo, obtener información sobre vacantes es la intención principal de uno de cada dos usuarios mientras que solo uno de cada diez accede a estas comunidades online buscando presentar candidaturas.

Se observa un funcionamiento de los grupos de empleo en Facebook articulado sobre una cultura colaborativa a través de la que se fomenta un uso compartido de los mismos a favor de un interés general -en este caso, la inserción laboral- y en detrimento de un empleo individualista. En este punto cabe matizar sin embargo que, en el caso hipotético de que con total seguridad el grupo de empleo fuese consultado por empresas que necesitasen personal, casi la totalidad de sus usuarios sí se decantaría por mostrar en público su perfil o candidatura profesional. De este modo, se abren posibilidades futuras en el uso de los grupos de empleo en Facebook, llamados a convertirse -si llegan a producirse las condiciones detalladas- en aplicaciones especializadas para la inserción laboral de sus usuarios a través de plataformas (los grupos) que, como se ha visto, permiten distribuciones geográficas -apostando por la proximidad- y ofrecen circunstancias apropiadas -multimedialidad, actualidad o interacción- para la presentación personal y la difusión de candidaturas profesionales en un entorno digital y compartido.

ICONO14 | Año 2015 Volumen $13 N^{\circ} 1$ | ISSN: 1697-8293 | DOI: ri14.v13i1.742 
Asimismo, tal y como se presuponía en la segunda hipótesis señalada, un grado de actualización frecuente -en este caso constante- y un uso diario por parte de sus miembros - uno de cada dos confiesa acceder al menos entre una y dos ocasiones al día- hacen que los grupos de empleo en Facebook puedan ser entendidos como herramientas de comunicación para el fomento de la inserción laboral. En este sentido, se ha podido conocer que el valor de actualidad, relacionado con la frecuencia de publicación de contenidos, no depende ni del ámbito de cobertura ni del número de usuarios con que cuente un determinado colectivo, como a priori se podía pensar. Por regla general, puede afirmarse que los grupos de temática laboral en Facebook son comunidades digitales en constante actualización, de empleo diario por parte de sus integrantes y con un índice de uso elevado.

Pero además de permitir confirmar la veracidad de las hipótesis de partida, esta investigación ha facilitado información relevante también en otros aspectos. Figura entre ellos el papel del administrador, cuya actividad parece relacionarse directamente con el índice de actualización del grupo que gestiona, pues es en los grupos con un índice de actualización más elevado donde el papel del creador o propietario es porcentualmente más importante. El tipo de fuentes empleadas es otra de las cuestiones sobre las que se han podido extraer datos relevantes. En este sentido, puede afirmarse que las comunidades de temática laboral en Facebook se nutren, para generar contenido en sus propios muros, de otras entidades de empleo. Asimismo, estas plataformas se presentan como espacios de usabilidad diversificada en la que la presencia de aplicaciones es constante y el índice de multimedialidad muy alto gracias al uso de imágenes, enlaces y documentos.

Los valores de usabilidad son, sin embargo, opuestos a los recogidos para el nivel de interactividad, caracterizado por índices bajos de apoyo a la información o de publicación de comentarios, inexistencia de comunicación entre los usuarios y los administradores de los grupos o consulta de información como uso casi preferente por parte de los integrantes.

En relación al perfil mayoritario de los usuarios integrados en este tipo de colectividades, el estudio realizado permite señalar que se trata de personas en situación de desempleo que buscan poner fin a esta situación laboral y que tienen 
a Internet como fuente de información principal para ello. Para uno de cada dos usuarios con estas características, un grupo de empleo es ya su fuente principal a la hora de buscar trabajo y tres de cada cuatro integrantes reconocen haber optado a vacantes conocidas mediante estas plataformas.

\subsection{Conclusiones}

De los datos derivados del análisis de contenido y de los cuestionarios realizados se puede concluir que en los grupos de empleo creados en Facebook se desarrolla una estrategia de comunicación para la inserción laboral que se articula en base a una cultura colaborativa. En ellos, sus usuarios priorizan la posibilidad de compartir información relacionada con vacantes sobe el uso de estos grupos como plataformas de promoción profesional. Asimismo, se ha constatado una recurrencia diaria por parte de la mayor parte de los miembros a estos grupos así como una actualización constante de contenidos, lo que permite describir a estas comunidades como colectivos activos que, fundamentalmente, a la hora de mantenerse actualizados dependen en buena medida de la labor de sus creadores, quienes los impulsan y dinamizan.

Por tanto, estas comunidades se presentan como aplicaciones activas respecto a la generación de contenidos pero con bajos niveles de participación. Se puede afirmar por tanto que en ellas no prima la interacción entre los usuarios -como sucede habitualmente en los entornos de redes sociales en los que se busca el contacto con otros amigos o seguidores- sino la colaboración a favor de un fin entre los que destaca, tal y como se ha demostrado, la ayuda a otros por medio de la difusión 0 consulta de información laboral.

En resumen, los grupos de empleo en Facebook se muestran como plataformas de comunicación para la inserción laboral, articuladas sobre una cultura colaborativa, satisfactorias para sus integrantes y recomendadas por ellos. Son, además, colectivos de actualización frecuente, usados a diario y en los que, pese a su alto índice de usabilidad, pesa más su utilización como fuente de consulta de información que su empleo como entorno interactivo. Se puede concluir, en definitiva, que en estos grupos se desarrolla entonces una estrategia de comunicación para la inserción laboral.

ICONO14 | Año 2015 Volumen $13 N^{\circ} 1$ | ISSN: 1697-8293 | DOI: ri14.v13i1.742 


\section{Notas}

[1] Ver http://investor.fb.com/releasedetail.cfm?ReleaseID=761090 (Fecha de último acceso: $31 / 07 / 2014)$

[2] Ver https://es-es.facebook.com/help/162866443847527/ (Fecha de último acceso: 31/07/2014)

[3] Ver https://www.facebook.com/groups/182379795160103/ (Fecha de último acceso: 25/07/2014)

[4] Ver https://www.facebook.com/groups/1394079800805956/ (Fecha de último acceso: 31/07/2014)

[5] Ver https://www.facebook.com/groups/182058195313274/ (Fecha de último acceso: 31/07/2014)

\section{Referencias}

Acosta V., G.L. (2010). Gestión de pasiones en las redes sociales. Un análisis del aplicativo Grupos en Facebook. Discurso y Sociedad, 6(4), 684-719. Recuperado el 10 de julio de 2014 de http://www.dissoc.org/ediciones/ v06n04/DS6\%284\%29Acosta.pdf

AIMC (2014). Navegantes en la Red - Encuesta AIMC a usuarios de Internet $16^{a}$. Madrid: Sersa. Recuperado el 11 de julio de 2014 de http://download.aimc. es/aimc/J5d8yq/macro2013.pdf

Arroyo-Vázquez, N. (2009). El uso profesional de las redes sociales. Anuario ThinkEPI, 3, 145-152.

Berlanga Fernández, I. (2013). El discurso en los medios digitales: principios de retórica clásica en las redes sociales, el caso de Facebook. (Tesis Doctoral, Universidad de Granada). Recuperado el 5 de agosto de 2014 de http://hdl. handle.net/10481/29796

Caldevilla Domínguez, D. (2010). Las Redes Sociales. Tipología, uso y consumo de las redes 2.0 en la sociedad digital actual. Documentación de las Ciencias de la Información, 33, 45-68. Recuperado el 18 de julio de 2014 de http:// revistas.ucm.es/index.php/DCIN/article/view/DCIN1010110045A

Castañeda, L. \& Gutiérrez, I. (2010). Redes Sociales y otros tejidos online para conectar personas. En Castañeda, L. (Coord.), Aprendizaje con Redes Sociales. Tejidos educativos en los nuevos entornos. Sevilla: MAD Eduforma.

Castells, M. (2000). Internet y la Sociedad Red. Conferencia de presentación

DOI: ri14.v13i1.742 | ISSN: 1697-8293 | Año 2015 Volumen 13 Nº 1 | ICONO14 
del Programa de Doctorado sobre la Sociedad de la Información y el conocimiento, Universitat Oberta de Catalunya. Recuperado el 10 de julio de 2014 de

http://instituto162.com.ar/wp-content/uploads/2014/04/INTERNET-Y-LASOCIEDAD-RED-Castells.pdf

Freire, J. (2009). Redes Sociales: ¿modelos organizativos o servicios digitales? El Profesional de la Información, 17(6), 585-588. doi:10.3145/epi.2008.nov.01 García Giménez, D. (2010). Redes sociales: posibilidades de Facebook para las bibliotecas públicas. BID Textos universitaris de biblioteconomía $i$ documentació, 24. Recuperado el 12 de julio de 2014 de http://bid. ub.edu/24/garcia1.htm

González, P. (2012). Facebook, plataforma para crear actividades cooperativas. Actas de las V Jornadas Didácticas del Instituto Cervantes de Mánchester, Universidad de Barbados, 29 de julio. Recuperado el 24 de julio de 2014 de http://cvc.cervantes.es/ensenanza/biblioteca_ele/publicaciones_centros/ PDF/manchester_2012/07_gonzalez.pdf

Hernández Sampieri, R. (5 a Ed).(2010). Metodología de la investigación. México: McGraw-Hill.

INE (2014). Encuesta de Población Activa (EPA). Recuperado el 7 de agosto de 2014 de

http://www.ine.es/inebaseDYN/epa30308/epa_inicio.htm\#

Llorens, F. \& Capdeferro, N. (2011). Posibilidades de la plataforma Facebook para el aprendizaje colaborativo en línea. Revista de Universidad y Sociedad del Conocimiento (RUSC), 8 (2), 31-45. Recuperado el 12 de julio de 2014 de http://journals.uoc.edu/index.php/rusc/article/view/v8n2-llorens-capdeferro/ v8n2-llorens-capdeferro-esp

Margaix-Arnal, D. (2008). Las bibliotecas universitarias y Facebook: cómo y por qué estar presentes. El profesional de la información, 17(6), 598-601. doi: 10.3145/epi.2008.nov.02

Marti i Puig, S. (2014). Los movimientos sociales. (Recurso formativo, Universidad de Salamanca). Recuperado el 13 de julio de 2014 de http://campus.usal. es/ dpublico/areacp/materiales/Losmovimientossociales.pdf

Narbona, J. (2012). El online communication model: un marco teórico para analizar la comunicación institucional en internet. Comunicazione della 
Chiesa: Volti, Persone, Storie (Atti del VIII Seminario Professionale sugli Uffici di Comunicazione della Chiesa), Roma, 16-18 abril.

Offe, C. (1996). Los nuevos movimientos sociales cuestionan los límites de la política institucional. En Offe C. (Ed). Partidos políticos y nuevos movimientos sociales (163-239). Madrid: Editorial Sistema.

Ros-Martín, M. (2009). Evolución de los servicios de Redes Sociales en Internet. El Profesional de la Información, 18(5), 552-558. doi: 10.3145/epi.2009. sep.10

The Coktail Analysis (2013). V Oleada. Observatorio de Redes Sociales. Informe Público de Resultados. Recuperado el 27 de julio de 2014 de http:// tcanalysis.com/blog/posts/el-70-de-los-usuarios-de-redes-sociales-semuestran-receptivos-a-la-presencia-de-marcas-en-este-entorno Zamora, M. (2006). Redes Sociales en Internet. (Publicación en blog). Recuperado el 10 de julio de 2014 de http://www.maestrosdelweb.com/editorial/ redessociales/

\section{Otras fuentes}

- http://www.alexa.com/siteinfo/facebook.com

- https://www.google.es/trends/explore\#q=facebook $\% 2 \mathrm{C} \% 20$ twitter\%2C\%20 google $\% 2 \mathrm{~B} \% 2 \mathrm{C} \% 20$ instagram $\% 2 \mathrm{C} \% 20$ linkedin

- http://investor.fb.com/releasedetail.cfm?ReleaseID=761090

- https://es-es.facebook.com/help/162866443847527/ 